\title{
ONE-SIDED DIVISION ABSOLUTE VALUED ALGEBRAS
}

\author{
Angej, Ronríglez Paiacios
}

\begin{abstract}
We develop a structure theory for left division absolute valued algebras which slows, among other things, that the norm of such an algebra comes from nu inner product. Moreover, we prove the existence of left division complete absolute valued algebras with left unit of arbitrary infinite hilbertian dimension and with the additional property that they lave no nonzero proper closed left ideals. Our construction involves results from the representation theory of the so called "Canonical Anticommutation Relations" in Quantum Mechanics. We also show that homomorphisms from complete normed algebrus into urbitrary absolute valued algebras are contractive, hence autonatically continuous.
\end{abstract}

\section{ln memoriam}

Este artículo ha sido escrito en homenaje a Pere Menal i Brufal. Acaso las matemáticas no sean el medio más apropiado para expresar el dolor por la muerte de este gran amigo asi como la adriración que su trabajo me merecía. Las matemáticas no obstante propiciaron una bella amistad y fueron credo y lenguaje corrún entre nosotros.

\section{Introduction}

A still unsolved old question is that of the nonassociative extension of the Gelfand-Mazur theorem, namely if any division normed (nonassociative) algebra must be finite-dimensional (which would inply dimension 1 in the complex case, and $1,2,4,8$ in the real one, by a theorem of $R$. Bot.t. and J. Milnor [6]). This problem was explicitly posed by F. B. Wright [27] in 1953, who in the same paper gave a partial affirmative answer proving that division absolute valued algebras are finite-dimensional. Another folklore partial positive result about this question is that outsided division complete normed complex algebras are isomorphic to the 
complex field (see [14]), the case of (two-sided) division noncomplete normed complex algebras as well as that of division (even complete) normed real algebras remaining open. In the converse direction, J. A. Cuenca [10] has recently given examples of infinite-dimensional one-sided division absolute valued algebras:over the field of real numbers.

Since the fact that one-sided division absolute valued complex algebras are isomorphic to the complex field can also be considered as folklore (see Proposition 2 in this paper), it seems to be reasonable to look for a structure theory of arbitrary one-sided division absolute valued real algebras, and in lact to provide such a structure theory is the main purpose of this paper.

To understand the pliflosophy of our work, it is suitable to take into account that, easily, lefi division absolute valued algebras are "isotopic" to left division absolute valued algebras with a left unit (sce Proposition 4). So the attention must be centered on these last algebras, and then the set (say $P$ ) of all left multiplication operators on such an algebra is a subspace of bounded linear operators on the normed space (say $X$ ) of the algebra containing the identity operator and satisfying

$$
\|T(x)\|=\|T\|\|x\|
$$

for all $T$ in $P$ and $x$ in $X$. We isolate this information in the first section of the paper, and we prove in Theorem 1 that, if $P$ is a subspace of bounded linear operators on any real normed space $X$ containing the identity operator (say $I$ ) and satisfying (*), then the operator norm on $P$ derives from an inmer product and, for $T$ in $P$ orthogonal to $I$, the equality

$$
T^{2}=-\|T\|^{2} Y
$$

holds. It follows that every clement in $P$ is an invertible operator or $X$ so, as a first consequence, absolute valued algebras with left unit are automatically left division algebras.

The content of the above referred Theorem 1 is stated in the paper in an equivalently reformulated form involving certain prehilbertian quadratic division-Jordan algebras with involution which, curiously, have been shown in $[20]$ to be the only "smooth normed" norlassociative cormmutative algebras. From this version of Theorem 1 we derive in Section 2 the main result in the paper (see Theorem 2) asserting that "unital *representations" of these Jordan algebras on their own pre-Hilbert spaces naturally give rise to (automatically left division) absolute valued algebras with left unit, and that by this constructive method all absolute valued algebras with left unit arise. This last assertion in Theorem 2 
means that the norm of an absolute valued algebra $A$ with loft unit $e$ comes from an inner product (. .) satisfying

$$
(a b \mid c)=-(b \mid a c) \text { and } a(a b)=-\|a\|^{2} b
$$

for all $a, b, c \operatorname{in} A$ with $a$ orthogonal to $e$.

In the third section we use Theoren 2, together with the basic facts about the so-called "canonical anticommutation relations" for quantum mechanics (which the reader nuy find in the first pages of $[8]$ ), to prove in Theorem 3 the existence of complete absolute valued algebras with left unit of arbitrary infinite hibertian dimension, and that such algebras can be chosen witli the additional property that they have no nonzero proper closed left ideals (note that every left division algebra has no non zelo proper right, ideals). Since small perturbations of the product of a left division complete absolute valued algebra give rise to new left division algebras (Proposition 7), the existence of a wide collection of infinite-dimensional left division nor-absolute-valued complete romed algcbras is asured. Let us emphasize also the existence of infinitedimensional complete normed algebras on which the operators of left and right multiplication by any nonzero element are surjective (a corsequence of Proposition 8).

The concluding section o[ the paper (Section 4) is devoted to prove the automalic continuity of homomorphisms from complete nomed algebras into absolute valued algebras. More perecisely, we show that such homomorphisms are contractive. This is perhaps the first nontrivial result involving arbitrary absolute valued algebras.

Though the axion $\|a b\|=\|a\|\|b\|$ seems to be very natural, the study of absolute valued algebras has received the attention of a relatively suall number of authors. Thus we are only aware of the papers (cited cronologically) by A. A. Albert [1] (1947) and [2], F. B. Wright [27], K. Urbanik and F. B. Wright [26], K. Urbanik [24] and [25], M. L. El-Mallah and A. Micali [19], and M. T. El-Mallah [15], [16], [17] and $[\mathbf{1 8}](1990)$. We emphasize the result in $[26](1960)$ asserting that $\mathbb{R}, C, M$, and $O$ are the only absolute valued algebras with unit (a fact that, as it will be explained in Remark 4(i), can be easily derived from Theorem 2). Also let us mention its easy consequence, previously proved in [27] and already mentioned at the beginning of this introduction, that division absolute valued algebras are finite-dimensional, as well as the relevant result in [19] showing that absolute valued algebras satisfying the identity $a(b a)=(a b) a$ also must be finite-dimensional. Examples of infinite-dimensional absolute valued algebras were known in the classical literaliure (see [26], [24], [3], and Remark 3(i)), but none of the algebras in these examples are one-sided division algebras. 1 hope our paper, 
as well as the almost simultaneous one by J. A. Cuenca $[10]$, could contribute as a revulsive to a subsequent flourishing development of the theory of absolute valued algebras.

\section{Subspaces of linear operators whose nonzero elements are multiples of isometries}

In this section we will deal with subspaces $P$ of bounded linear operators on a real normed space $X$ containing the identity operator on $X$ and with the property that

$$
\|T(x)\|=\|T\|\|x\|
$$

for all $x$ in $X$ and all $T$ in $P$. The results obtained here will become the main tools for the proof in the next section of the structure theory for one-sided division absolute valued algebras. In fact, we will prove that the operator norm on subspaces of operators as considered above derives from an inner product. It will also turn out that these subspaces are actually quadratic Jordan algebras of operators whose nonzero elements are invertible, so that they correspond with some abstract mathematical models previously considered in the answer to other problems and that are closely related with the "canonical anticommutation relations" of quantum mechanics. In fact, the possibility of representing the canonical anticommutation relations by means of bounded linear operators on Hilbert spaces will allow us to prove in Section 3 the existence of infinitedimensional one-sided division absolute valued algebras with additional properties.

We begin our argument with the following (perhaps well-known) lemma. For a normed space $X, B L(X)$ will denote the associative normed algebra of all bounded linear operators on $X$.

Lemma 1. Let $X$ be a Banach space, and $T: X \rightarrow X$ be a lineat isometry which is not onto. Then the open unit ball in $B L(X)$ with center $T$ and radius 1 contains only operators with closed range and which are one-to-one but not onto.

Proof: Recall that a linear operator $T$ on $X$ is said to be bounded below (by $m>0$ ) if $\|T(x)\| \geq m\|x\|$ for all $x$ in $X$. Since, for $T$ in $B L(X), T$ is one-to-one with closed range if and only if $T$ is bounded below, the lemma will follow from the more general result, we will prove here, that, if $T$ is bounded below by $m$ and is not onto, then the open ball in $B L(X)$ with center $T$ and radius $m$ contains only bounded below 
operators which are not onto. Easily, for such a $T$ and any $S$ in $B L(X)$ with $\|T \cdots S\|<m, S$ is bounded below by $m-.\|T-S\|$. If actually $\|T-S\|<\frac{m}{2}$ and $S$ is surjective, we have tlat $S$ is invertible in $B L(X)$ with

$$
\text { . }\left\|S^{-1}\right\| \leq \frac{1}{m-\|T-S\|}<\frac{2}{m} \text {, so }\|T-S\|\left(<\frac{m}{2}\right)<\left\|S^{-1}\right\|^{-1} \text {, }
$$

and so $T$ is invertible in $B L(X)[4$, Theorem 2.11 , which is a contradiction. In this way we have proved that the open ball in $B L(X)$ with center $T$ and radius $\frac{m}{2}$ contains only bounded below operators wich are not onto. It follows that the set

$$
\Omega:=\{R, B L(X): R \text { bounded below and not onto }\}
$$

is open. Now the open ball in $B L(X)$ with center $T$ and radius $m$ is a connected topological space which is the disjoint union of its interscctions with $\Omega$ and with the set of invertible elements of $B L(X)$. Since these intersections are open and the first one is nonempty, it follows that the refered ball is contaned in $\Omega$, as required.

At this time we recall some concepts suitable for a reasonably clear statement of our main result in this section. A Jordan culgebra is a commutative algebra (say $J$ ) satisfying the "Jordan identity", namely

$$
x^{2} \cdot(y, x)=\left(x^{2} \cdot y\right) \cdot x
$$

for all $x, y$ in $J$. The most easy examples of Jordan algebras are the socalled "Jordan subalgebras of associative algebrus", namely subspaces of an associative algebra which are closed under the "Jordan product"

$$
a \cdot b:=\frac{1}{2}(a b+b a)
$$

where the elssociative product has been denoted as usually by juxtaposition. In case the associative algebra is the one of all linear operators on a vector space $X$, such Jordan subalgebras are called "Jordau algebras of operators on $X$ ". An algebra is said to be quindratic if it has a unit 1 arid, for every $x$ in the algebra, there exist $\lambda$ and $\mu$ in the base field such that; $x^{2}+\lambda x+\mu \mathbf{1}=0$. A normed algebra is a real or complex algebra whose vector space is a normed space with respect to a norm $\|$. II satisfying $\|x y\| \leq\|x\|\|y\|$ for all $x, y$ in the algebra. A wnital nowned algebra is a nomed algebra with urit; 1 satisfying $\|I\|=1$. Finally a smooth normed elgebra is a unital normed algebra whose unit is a smooth point of its closed unit ball. All assertions in the following proposition, that are not of straighforward verification, are consequences of $[20$, Theorem 27] (sec also [22, Section 2]). 
Proposition 1. If $H$ is a real pre-Hilbert space and we consider on $\mathbb{R} 1 \oplus H$ the product given by

$$
(\lambda \mathbf{1}+\eta) \cdot(\mu \mathbf{1}+\xi):=(\lambda \mu-(\eta \mid \xi)) \mathbf{1}+(\lambda \xi+\mu \eta)
$$

for all $\lambda, \mu$ in $\mathbb{R}$ and $\eta, \xi$ in $H$, then $\mathbb{R} 1 \oplus H$ becomes a quadratic Jordan algebra over $\mathbb{R}$ which, endowed with the pre-Hilbert norm

$$
\|\lambda \mathbf{1}+\eta\|:=\left(\lambda^{2}+\|\eta\|^{2}\right)^{1 / 2}
$$

is a smooth normed algebra. Moreover, every real smooth normed commutative algebra arises in this way.

We will refer to the normed algebras described in the above proposition as the "smooth normed Jordan algebras".

Remarks 1. i) An element $x$ in a Jordan algebra $J$ with unit is said to be invertible if there exists $y$ in $J$ with $x \cdot y=1$ and $x \cdot y^{2}=y$ (see [13, Definition I.11.5]). A division-Jordan algebra is a Jordan algebra with unit whose nonzero elements are invertible. It is easy to see that all smooth normed Jordan algebras are division-Jordan algebras, so they are real normed division-Jordan algebras under any algebra norm (whicht must be greater than the canonical pre-Hilbert norm because this last norm equals the spectral radius). It was proved in [14] that no more real normed division-Jordan algebras exist.

ii) It is well-known that if $A$ is an associative algebra with unit 1 and $J$ is a Jordan subalgebra of $A$ with $1 \in J$, then an element $x$ in $J$ is invertible in $J$ in the above sense if and only if $x$ is invertible in $A$ in the usual sense and its associative inverse lies in $J[13, \mathrm{p} .51]$. In the particular case of $J$ being (isomorphic to) one of the smooth normed Jordan algebras, this fact is almost obvious. For, if for $x=\lambda \mathbf{1}+\eta$ in $J=R 1 \oplus H$ we donote by $x^{*}$ the element in $J$ given by $x^{*}:=\lambda 1-\eta$, denoting by yuxtaposition the associative product of $A$ we have

$$
x^{*} x=x x^{*}=\lambda^{2} \mathbf{1}-\eta^{2}=\lambda^{2} \mathbf{1}-\eta \cdot \eta=\left(\lambda^{2}+\|\eta\|^{2}\right) \mathbf{1}=\|x\|^{2} \mathbf{1},
$$

so every nonzero $x$ in $J$ is invertible in $A$ in the associative sense, with associative inverse equal to $\|x\|^{-2} x^{*}$ (which of course lies in $J$ ).

iii) The normed space of a smooth normed Jordan algebra is certainly a nonzero real pre-Hilbert space, and, given any nonzero real pre-Hilbcrt space $K$, up to isometric isomorphisms there is a unique smooth normed Jordan algebra whose pre-Hilbert space is $K$. This is so because, chosing any norm-one element $u$ in $K$ and denoting by $H$ the orthogonal complement of $\mathbb{R} u$ in $K, H$ is the only pre-Hilbert space satisfying $K=\mathbb{R} u \oplus^{l_{z}} H$, 
so there is a unique conmutative product on the normed space of $K$ converting it in a smooth normed Jordan algebra whose unit is $u$. Moreover, the choice of the norm-one element $u$ in $K$ is irrelevant thanks to the "rotation property" of pre-Hilbert spaces, namely any norm-one element can be carried into another by means of a suitable surjective linear isometry. Indeed, this is clear for dimension 1 or 2 , and, in the remaining case, if $u$ and $z$ are nom-one elements in $K$ and if we denote by $L$ the linear bull of $\{u, v\}$ as well as by $p$ a linear isometry from $L$ onto $L$ with $\varphi(u)=v$, then the mapping

$$
\phi: l+l^{\perp} \longrightarrow \varphi(l)+l^{\perp}
$$

from $K=L \oplus L^{1}$ into $K$ is a surjective linear isometry satisfying $\phi(u)=$ v. As a consequence, given a cardirial mmber $\aleph$, there exists a unique smooth complete nomed Jordan algebra with hilbertian dimension equal to $k$.

Now we state and prove the main result in this section.

Theorem 1. Let $X$ be a nonzero real normed space, and $P$ be a subspace of bounded linenr operators on $X$ containing the itlentity operator and satisfying

$$
\|T(x)\|=\|T\|\|x\|
$$

for all $T$ in $P$ and all $x$ in $X$. Then $P$ is a Jordan alyebrn of operalors on $X$ which, endoved with the operator norm, is isometrically isomorphic to some of the sriooth normed Jordan algebris:

Proof: In a first step we reduce the proof to the particular case of $X$ being a Banach space. To this end, consider the completion $\bar{X}$ of $X$ and, for $T$ in $B L(X)$, let $\bar{T}$ denote the unique element in $B L(\bar{X})$ which extends $T$. Then the isometric homomorphism $T \rightarrow \ddot{T}$ from the associative normed algebra $B L(X)$ into the Banach algebra $B L(\bar{X})$ maps $P$ onto a subspace (say $\bar{P}$ ) of $B L(\bar{X})$ which clearly inherits the properties of $P: \bar{P}$ is a subspatce of bounded linear operators on $\bar{X}$ containing the identity operator on $\bar{X}$ and satisfies

$$
\|S(y)\|=\|S\|\|y\|
$$

for all $S$ in $\bar{P}$ and all $y$ in $\bar{X}$. In this way, if the theorem is true in the complete case, the information it gives about $\bar{P}$ is easily transfered to $P$.

In a second stcp we assume $X$ to be a Banach space and we show that then every nonzero element in $P$ is invertible in $B L(X)$. This fact being clear if the dimension of $P$ is one, assume $\operatorname{dim}(P) \geq 2$, so that $P \backslash\{0\}$ 
with the topology of the operator norm is a connected topological space in which the subset

$$
\{T \in P \backslash\{0\}: T \text { is invertible in } B L(X)\}
$$

is certainly open (see [4, Theorem 2.11]) and nonempty. Now this second step is concluded by verifying that the complementary subset

$$
\Omega:=\{T \in P \backslash\{0\}: T \text { is not invertible in } B L(X)\}
$$

is also open in $P \backslash\{0\}$. But, if $T$ is in $\Omega,\|T\|^{-1} T$ is a linear isometry from $X$ into $X$ which is not onto and so, by Lemma 1, the open ball in $P \backslash\{0\}$ with center $T$ and radius $\|T\|$ is contained in $\Omega$, hence $\Omega$ is open in $P \backslash\{0\}$, as required. Since in what follows complex methods will be applied, it is suitable to summarize our situation in the following way. $P$ is a real subspace of a unital complex (associative) Banach algebra (in our case, the normed complexification of $B L(X)$ [4, Proposition 13.3]) that will be denoted by $A$, the unit of $A$ lies in $P$, and every nonzero element $T$ in $P$ is invertible in $A$ with $\left\|T^{-1}\right\|=\|T\|^{-1}$. Note that this last property implics

$$
|z|=\|T\|
$$

for all $T$ in $P$ and all $z$ in the spectrum of $T$ relative to $A$.

The third step in our proof consists in showing that, for $T$ in $P \backslash R 1$, the real linear hull of $\{1, T\}$ with the restriction of the norm of $A$ is a copy of the euclidean space $\mathbb{R}^{2}$. Chose $z$ in the spectrum of $T$ relative to $A$ and write $\lambda:=\operatorname{Re}(z)$ and $S:=\|T-\lambda \mathbf{1}\|^{-1}(T-\lambda \mathbf{1})$, so that $S$ lies in $P,\|S\|=I$, and the spectrum of $S$ contains a number of the form $i \epsilon$ for some $\epsilon$ in $\mathbb{R}$. Then, for arbitrary $\alpha, \beta$ in $\mathbb{R}, \alpha+i \epsilon \beta$ lies in the spectrum of $\alpha 1+\beta S$ so, by $(*)$, we have

$$
\begin{aligned}
\|\alpha 1+\beta S\|^{2} & =|\alpha+i \epsilon \beta|^{2}=\alpha^{2}+\epsilon^{2} \beta^{2}= \\
& =\alpha^{2}+|i \epsilon|^{2} \beta^{2}=\alpha^{2}+\|S\|^{2} \beta^{2}=\alpha^{2}+\beta^{2},
\end{aligned}
$$

so that $(\alpha, \beta) \rightarrow \alpha 1+\beta S$ is a linear isometry from the euclidean space $\mathbb{R}^{2}$ onto $\operatorname{Lin}_{\mathbb{R}}\left\{1, T^{\top}\right\}$.

Our concluding step of the proof of the theorem will show as desired that $P$ is a real Jordan subalgebra of $A$ isometrically isomorphic to one of the smooth normed Jordan algebras. The consequence that the operator norm on $P$ derives from an inner product can be easily obtained from the above step applied to the subspaces of $B L(X)$ of the form $\left\{S^{-1} T: T \in P\right\}$ with $S$ any nonzero fixed element in $P$; but in fact the 
prehilbertian nature of $P$ will be reencountered in what follows jointly with the remaining part of the information. We will use some concepts and resuits from the theory of numerical ranges. Thus, recall that an elernent $a$ in $A$ is said to be hermitian if $f(a)$ lies in for all $f$ in the dual Banach space of $A$ with $\|f\|=f(1)=1$. Clearly, the set of all hermitian elements in $A$ is a real subspace of $A$ so, if we denote by $H$ the set of those $S$ in $P$ such that $i S$ is an hermitian element in $A, H$ is a subspace of $P$, and we claim $P=\mathbb{R} 1 \oplus^{l_{2}} \mathrm{H}$. Indeed, obviously $\mathbb{P 1} \cap H=0$ and, by the third step of the proof, every $T$ in $P$ is of the form $\lambda 1+S$ for suitable $\lambda$ in $R$ and $S$ in $P$ with $\|\alpha 1+\beta S\|^{2}=\alpha^{2}+\|S\|^{2} \beta^{2}$ for all $\alpha, \beta$ in $\mathbb{R}$, an equality that implies

$$
\|\lambda \mathbf{1}+S\|^{2}=\lambda^{2}+\|S\|^{2} \text { and } \lim _{\beta \in \mathbb{A} \backslash\{0\}, \beta \rightarrow 0} \frac{\|\mathbf{1}+\beta S\|-1}{\beta}=0
$$

so $i S$ is hermitian in $A$ [4, Theorem 10.10], and so $S$ lies in $H$, concluding the proof of the claim. A Theorem by B. Bollobas (see [ $\mathbf{5}$, Theorem 26.7]) asserts that, if $a$ is an invertible hermitian element of a unital complex Banach algebra with $\|a\|=\left\|a^{-1}\right\|=1$, then $a^{2}=1$. This applies in particular to clements of the form $\frac{i}{\|S\|} S$ with $S$ in $H \backslash\{0\}$ to obtain

$$
S^{2}=-\|S\|^{2} 1
$$

for all $S$ in $H$, hence the restriction of the norm of $A$ to $H$ comes from an inner product $(. \mid$.). In passing from quadratic mappings to associated symmetric bilinear mappings, we find

$$
S . T=-(S \mid T) 1
$$

for all $S, T$ in $H$. Finally, for every $\lambda \mathrm{I}+S$ and $\mu \mathrm{I}+T$ in $P=\mathbf{R} \mathbf{1} \oplus^{l_{2}} H$, we have

$$
(\lambda 1+S) \cdot(\mu 1+T)=(\lambda \mu-(S \mid T)) x+\lambda T+\mu S
$$

so certainly $P$ is a real Jordan subalgebra of $A$ which is a materialization of a smooth normed Jordan algebra.

Corollary 1. Let $X$ be a nonzero real normed space, $P$ be a subspace of $B L(X)$ satisfying

$$
\|T(x)\|=\|T\|\|x\|
$$

for all $T$ in $P$ and all $x$ in $X$, and assume that some element in $P$ is invertible (with inverse possibly outside of $P$ ). Then the operator norm. 
on $P$ derives from an inner product, and all nonzero elements in $P$ are invertible.

Proof: If the identity operator on $X$ lies in $P$, the corollary is a direct consequence of the theorem, taking into account of course the information about smooth nomed Jordan algebras previously collected in Proposition 1 and Remark 1.(ii). In the general case, since there exists some $S$ in $P$ which is an invertible operator with $\|S\|-\left\|S^{-1}\right\|=1$, the mapping: $T \rightarrow S^{-1} T$ is an invertible-preserving linear isometry from $P$ onto a subspace. (say $Q$ ) of $B L(X)$ with the same properties that of $P$ but which contains the identity operator on $X$. Now it is clear how the properties of $Q$, of being a pre-Hilbert space and having invertible all its nonzero elements, can be transfered to $P$.

Remarks 2. i) Let $X$ and $\dot{P}$ be as in the corollary. If $X$ is actually a Banach space, the assertion that all nonzero elements of $P$ are invertible operators is an easy consequence of Lemma 1 (recall the second step in the proof of 'Theorem 1). However, the reader can verify that the reduction method in the first step of Theorem 1 alone does not allow to transfer this information to the general noncomplete case (the point of difficulty is that the extension by continuity of an element $T$ in $B L(X)$ to the completion of $X$ may be invertible, while $T$ is not). Therefore, it seems that the proof of the above commented assertion in the noncomplete case needs the whole of Theorem 1.

ii) Again, under the assumptions of Corollary 1 and with the notation there, one can ask about the algebraic siructure of $P$. With the method in the proof of the corollary and the information given by Theorem 1, one obtains easily that $T S^{-1} T$ lies in $P$ whenever $S$ and $T$ are in $P$ with $S \neq 0$. More precisely, for each norm-one element $S$ in $P: P$ with the operator norm and product $\odot$ given by

$$
R \odot T:=\frac{1}{2}\left(R S^{-1} T+T S^{-1} R\right)
$$

is a smooth normed Jordan algebra. Although these facts invite to think that $P$ must be a "Jordan triple system of operators" on $X$ (subspaces of linear operators on $X$ containing $T S T$ whenever $S$ and $T$ are in the subspace), this is not the case in general, as shown by the followirg example. Consider the division associative algebra $\boldsymbol{H}$ of real quaternions with its usual absolute value as norm, take $z$ in $\mathrm{H}^{-1}$ with $z^{2} \notin \mathbb{R}$; and for $y$ in H let $T_{y}$ denote the operator on $B$ given by $T_{y}(x):=y x z$ for all $x$ in $H$. Then, if $X$ denotes the normed space of $H$ and we write $P:=\left\{T_{y}: y \in \mathbb{H}\right\}, P$ is a subspace of $B L(X)$ satisfying the assumptions in Corollary 1. If $P$ was a Jordan triple system of operators on $X$, we 
would have $T_{1}^{3} \in P$, giving the existence of some $y$ in $\mathbf{H}$ with $x z^{2}=y x$ for all $x$ in $H$ (implying, with $x=1, z^{2}=y$ ), so $z^{2}$ would be in the centre of $\mathbf{H}$ which equals $\mathbf{R} \mathbf{1}$, contrary to the choice of $z$. Subspaces $P$ of associative algebras with unit satisfying that all its nonzero elements are invertible and that $T S^{-1} T$ lies in $P$ whenever $S$ and $T$ are in $P$ with $S \neq 0$ seem to appear here for the first time in a natural way. It might be interesting to explore abstractly the structure of such subspaces.

In concluding this section, let us consider spaces of linear operators acting on complex normed spaces whose nonzero elements are multiples of isometries and containing some invertible element. The result we obtain in this case is very obstructive, it follows easily from Corollary 1 but, since contrarily to what has been asserted in Remark 1(i) in relation with Corollary 1 the full strength of Theorem 1 now is not needed, we give a more direct proof.

Corollary 2. Let $X$ be a nonzero complex normed space, $P$ be a (complex) subspace of $B L(X)$ sulisfying

$$
\|T(x)\|=\|T\|\|x\|
$$

for all $T$ in $P$ and all $x$ in $X$, and assume that there exists some invertible element in $P$. Then $P$ is one-dimensional.

Proof: Since the extension by continuity to the completion of $X$ of an invertible linear isometry on $X$ is also an invertible linear isometry, the set of all extensions by continuity to the completion of $X$ of the elements of $P$ inherits all the properties of $P$, hence we may assume that $X$ is actually a Banach space. Then, as in the proof of the second step of Theorem 1, Lemma 1 gives easily that every ronzero element in $P$ is invertible (even the separate consideration there of the case $\operatorname{dim}(P)=1$ is now unnecessary). Finally chose $S$ in $P \backslash\{0\}$, let $T$ be any element in $P$, and $z$ be in the spectrum of $T S^{-1}$ relative to $B L(X)$. Then $T-z S=\left(T S^{-1}-z 1\right) S$ is a noninvertible element in $P$, hence equal to zero. It follows that $P=\mathrm{CS}$.

\section{Structure of one-sided division absolute valued algebras}

By a left division algebra we mean a nonzero algebra (say $A$ ) with the property that, whenever $a$ and $b$ are in $A$ with $a \neq 0$, there exists a unique $x$ in $A$ satisfying $a x=b$. Of course we can consider the analogous concept of right division algebra, and we say that $A$ is a division algebra 
if it is at the same time a left division and. a right division algebra.

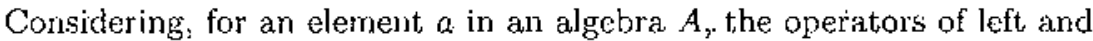
right multiplication by $a$ on $A$ denoted respectively by $L_{a}$ and $R_{a 1}$, it is clear that $A$ is a left (resp.: right) division algebra if and only if, for every nonzero $a$ in $A$, the operator $L_{a}$ ('resp.: $R_{a}$ ) is bijective.

Since clearly one-sided division algebras have no nonzero zero-divisors, it follows that a finite-dimensional algebra is a left division algebra iff it is a right division algebra, iff it has no nonzero zero-divisors.

Another relevant case in which one-sided division implies division is the associative one. Even in this case we reencounter the concept of a division associative algebra in its nost familiar sense, namely the associative algebra under consideration has a unit and all its nonzero elements are invertible. To see this, let $A$ be a left division associative algebra, chose $b$ in $A \backslash\{0\}$, and write $e:=L_{b}^{-1}(b)$. By the associativity of $A$, for every $x$ in $A, R_{x}$ commutes with $L_{b}$ so also with $L_{b}^{-1}$, and therefore we have

$$
e x=L_{b}^{-1}(b) x=R_{x} L_{b}^{-1}(b)=L_{b}^{-1} R_{x}(b)=L_{b}^{-1}(b x)=L_{b}^{-1} L_{b}(x)=x
$$

so that $e$ is a left unit for $A$. Then, using again the associativity of $A$ we obtain $(x e-x) A=0$, so $x e=x$ (since $A$ is nonzero and has no nonzero zero-divisors), hence $e$ is actually a (two-sided) unit for $A$. Since $A$ is a left division algebra, for every $a$ in $A \backslash\{0\}$ there is a inique $c$ in $A$ with $a c=e$, but, since then $a(c a-e)=0$, it follows that also $c a=e$, and therefore $a$ is invertible in $A$ with $a^{-1}=c$. Now, for every $a$ in $A \backslash\{0\}$, the operator $R_{a}$ is bijective (with inverse mapping equal to $R_{a^{-1}}$ ).

Recall that an absolute valued algebro is a nonzero real or complex algebra whose vector space is a normed space with respect to a norm $\|$. $\|$ satisfying $\|x y\|=\|x\|\|y\|$ for all $x, y$ in the algebra. The unsolved general question if every division normed algebra is finite-dimensional has an affirmative answer in' the particular cáse of absolute valued algebras. 'This is a well-known fact first proved by B. Wright [27]. Today it, follows easily from the celebrated Albert-Urbanik-Wright theorem [26], asserting that the orily absolute valued algebras with unit are $\mathbb{R}, \mathbb{C}, \mathbb{H}$ and $\mathbb{O}$ (the algebra of Cayley numbers), together with a standard argument of "isotopy" due to A. Albert [1]. In the next two propositions we state this result together with some improvements following from Corollaries 1 and 2 of the previous. section.

Proposition 2. For a complex absolute valued algebra $A$ the following assertions are equivalent:

i) There exists a in $A$ such that $L_{a}$ is an invertible operator on $A$.

ii) $A$ is isomorphic to the complex field. 
iii) $A$ is finite-dimensional.

iv) $A$ is a dinision algebra.

v) $A$ is a left division algebra.

Proof: Since the implications (ii) $\Rightarrow(i i i) \Rightarrow(i v) \Rightarrow(v) \Rightarrow$ (i) are clear (for $(i i i) \Rightarrow(i v)$ note that every alssolute valued algebra has no nonzero zero-divisors), it only remains to prove that (i) implies (ii). But it follows from the assumption $(i)$ and the fact that $A$ is a complex absolute valued algebra that the set $P:=\left\{L_{x: c}: x \in A\right\}$ is a subspace of bounded linear operatiors on the normed space of $A$ satisfying the requirements in Corollary 2 . Hence $P$ is one-dimensional and, since the mapping $x \rightarrow L_{x}$ is one-to-one, also $A$ is one-dimensional. Finally note that, any one-dimensional algebra with nonzero product is isomorphic to the base ficld.

The result characterizing division absolute valued algebras over $\mathbb{R}$ involves a peculiar concept of isotopy already used in the treatment of other problems in relation with absolute valued algebras. T'wo absolute valued algebras $A$ and $B$ are said to be isolopic if there exist linear isometries $\varphi_{1}, \varphi_{2}, \varphi_{3}$ from $A$ onto $B$ satisfying

$$
\varphi_{1}(x y)=\varphi_{2}(x) \varphi_{3}(y)
$$

for all $x, y$ in $A$. This notion is molivated by the obvious fact that, given an absolute valued algebra $A$ and arbitrary linear isometries $\varphi_{1}, \varphi_{2}, \varphi_{3}$ from $A$ onto $A$, the normed space of $A$ with the new product $\odot$ given by

$$
x \odot y:=\varphi_{1}^{-1}\left(\varphi_{2}(x) \varphi_{3}(y)\right)
$$

becomes also an absolute valued algebra. For our purposes, it should be noted that isotopies preserve left, right, and two-sided clivision.

Proposition 3. For a real absolute valued algebra $A$ the following assertions are equivalent:

i) There exist $a$ and $b$ in $A$ such that $L_{a}$ and $R_{b}$ are invertible op)erators on $A$.

ii) A is isotopic to $\mathbb{R}, \mathbb{C}, \mathbf{H}$, or $\mathbf{O}$.

iii) $A$ is finitc-dimensional.

iv) $A$ is a division algebra.

Proof: As in the proof of Proposition 2, it is enough to show that (i) implies ( $i i$ ). So let us assume that (i) holds, and consider the subspaces $P$ and $Q$ of bounded linear operators on the normed space of $A$ given by

$$
P:=\left\{L_{x}: x \in A\right\} \text { and } Q:=\left\{R_{x}: x \in A\right\} .
$$


By Corollary 1, every nonzero element in any of these subspaces is invertible, and then, chosing $c$ in $A$ with $\|c\|=1$, the normed space of $A$ with new product $\odot$ defincd by

$$
x \odot y:=R_{c}^{-1}(x) L_{c}^{-1}(y)
$$

becomes an absolute valued algebra with unit (equal to $c^{2}$ ). The above referred Albert-Urbanik-Wright theorem gives that this last algebra must be equal to $\mathbb{R}, \mathbb{C}, \mathbb{H}$, or $\mathbb{O}$. Hence the given absolute valued algebra $A$ is isotopic to one of these four algebras, as desired.

Remarks 3. i) Propositions 2 and 3 are nontrivial, because of the existence of real or complex infinite-dirnensional absolute valued algebras (see [26], [24], and [3]). The easiest examples of such algebras we know can be constructed in the following way. Consider an infinite set $U$, so that there exists a one-to-one mapping $\vartheta$ from $U \times U$ into $U$, let $A$ denote the free real or complex vector space generated by $U$ with bilinear product determined on the generators by

$$
u v:=\vartheta(u, v)
$$

for all $u, v$ in $U$, and let $\|$. $\|$ be the norm on $A$ given by either

$$
\left\|\sum_{u \in U} \lambda_{t} u\right\|:=\left(\sum_{u \in U}\left|\lambda_{u}\right|^{p}\right)^{1 / p}
$$

( $p$ any real number with $p \geq 1$ ), or

$$
\left\|\sum_{u \in U} \lambda_{u t} u\right\|:=\sup \left\{\left|\lambda_{u}\right|: u \in U\right\} .
$$

Then it is straighforward to verify that $A$ is an absolute valued algebra. Since completions of absolute valued algebras are absolute valued algebras, it follows that the classical Banach spaces $l_{p}(U)$ and $c_{0}(U)$ can be structured as absolute valued algebras. As a consequence, the existence of complete absolute valued algebras with nonrefiexive Banach space is guarantced.

ii) As it was anmounced in the introduction of [9], a relevant consequence of the preceding remark is that the free real or complex nonassociative algebra generated by any set is an absolute valued algebra under many pairwise nonequivalent norms. For, if $A$ denotes the free nonassociative algebra generated by an arbitrary set $V, A$ is nothing but the free vector space generated by the set $U$ of all "nonassociative words" 
with entries in $V$ with product the extension by bilinearity of the formal juxtaposition of the elements in $U$. Since pressing to juxtaposition of nonassociative words is a one-to-one nupping (see [13, Lemma in p. 24]), the preceding remark applies successfuly. The inclusion of this remark here is at courtesy of M. Cabrera.

iii) Infinite-dimensional absolute valued algebras cannot, satisfy any familiar identity like associativity or commutativity. While the result coucerning associativity is a consequence of the Gelfand-MazurKaplansky theorem on associative normed algebras with no nonzero zero-topological-divisoss, the one concerning commutativity is due to K. Urbanik and F. B. Wright [26]. Botll results are contained in the one by M. L. El-Mallah and A. Micali [19] asserting that an absolute valued algebra satisfying the identity $x(y x)=(x y) x$ is finite-dimensionat.

While by Proposition 2 the complex field is the only one-sided division absolute valued complex algebra, Proposition 3 does not prevent the existence of infinite-dimensional one-sided division absolute valued real algebras. The next proposition (a part of which is of folklore nature) and theorem (whose proof has been prejared in Section 1) will provide a structure theory for one-sided division absolute valued real algcbras, which will later inspire the construction of infinite-dimensional examples of such algebras. From now on, we tacithy assume that all vector spaces ant algebras are real.

Proposition 4. For an absolute valued algebra $A$ the following assertions are equivalent:

i) There exists a in $A$ such that $L_{a}$ is an invertible operator on $A$.

ii) $A$ is isotopic to an absolute valued algebra with left unit.

iii) $A$ is a left division algebra.

Proof: $(i) \Rightarrow$ (ii) We may assume that the element $a$ in $A$ such that $L_{a}$ is invertible has norm one, and then the normed space of $A$ with new product $\odot$ defined by

$$
x \odot y:=L_{n}^{-1}(x) L_{a}^{-1}(y)
$$

is an absolute valued algebra with lef unit. (cqual to $a^{2}$ ) which is isotopic to $A$.

(ii) $\Rightarrow$ (iii) Since isotopies prescrve left division, we may assume that $A$ has a left unit, and then the subspace $P$ of bounded linear operators on the normed space of $A$ given by $P:=\left\{L_{x}: x \in A\right\}$ satisfies the assimptions of Corollary 1 (actiually it satisfies even the requirements in Theorem 1), hence all its nonzero elements are invertible, that is $A$ is a left division algebra. 
(iii) $\Rightarrow(i)$ This is trivial.

An almost direct consequence of the implication $(i) \Rightarrow$ (iii) in the above proposition is the following

Corollary 3. The completion of a left division absolute valued algebra is a left division absolute valued algebra.

In view of Proposition 4, to have a satisfactory theory for left division absolute valued algebras it is enough to study (automatically left division) absolute valued algebras with left unit. To this end it is useful to introduce some additional terminology. Given a Jordan algebra $J$ and a vector space $X$, a representation of $J$ on $X$ will mean an homomorphism (say $\psi$ ) from $J$ onto a Jordan algebra of operators on $X$. If $J$ has a unit 1 and $\psi(1)$ equals the identity operator on $X$, the representation $\psi$ will be called unital. If $X$ is a pre-Hilbert space, $*$ is an algebra involution on $J$, and the representation $\psi$ satisfies

$$
(\psi(x)(\eta) \mid \xi)=\left(\eta \mid \psi\left(x^{*}\right)(\xi)\right)
$$

for all $x$ in $J$ and all $\eta, \xi$ in $X$, then we will say that $\psi$ is $a *$ representation. When $J$ and $X$ are normed, the representation $\psi$ will be called isometric (resp.: contractive) if, for all $x$ in $J$, the linear operator $\psi(x)$ on $X$ is bounded with $\|\psi(x)\|=\|x\|$ (resp.: $\|\psi(x)\| \leq\|x\|$ ). From now on every smooth normed Jordan algebra $J=\mathbb{R} 1 \oplus H$ will be considered as algebra with involution $*$ defined by

$$
(\lambda \mathbf{1}+\eta)^{*}:=\lambda \mathbf{1}-\eta
$$

This involution can be intrinsically characterized as the only algebra involution $*$ in $J$ such that, for every $x$ in $J, x+x^{*}$ and $x \cdot x^{*}$ lie in $\mathbb{R} 1$.

Lemma 2. Let $J$ be a smooth normed Jordan algebra, $K$ a nonzero pre-Hilbert space, and $\psi$ be a unital representation of $J$ on $K$. Then the following assertions are equivalent:

i) $\psi$ is a *-representation.

ii) $\|\psi(x)(k)\|=\|x\|\|k\|$ for all $x$ in $J$ and $k$ in $K$.

iii) $\psi$ is isometric.

iv) $\psi$ is contractive.

Proof: $(i) \Rightarrow(i i)$ Being $J$ a simple Jordan algebra and $\psi$ a unital representation, the range of $\psi$ is a Jordan subalgebra of the associative algebra $L(K)$, of all linear operators on the vector space of $K$, isomorphic 
to $J$ and containing the unit of $L(K)$ (namely, the identity operator on $J$, which will be denoted by $I$ ), hence by Remark 1 (ii) we have

$$
\psi\left(x^{*}\right) \psi(x)=\|x\|^{2} I
$$

for all $x$ in $J$. Therefore; from the assumption (i), we obtain for arbitrary $k$ in $K$

$$
\begin{aligned}
\| \psi(x)(k) !^{2} & =(\psi(x)(k) \mid \psi(x)(k))= \\
& \left.=\left(k \mid \psi\left(x^{*}\right) \psi(x)(k)\right)=(k]\|x\|^{2} k\right)=\|x\|^{2}\|k\|^{2} .
\end{aligned}
$$

(ii) $\Rightarrow($ iii $) \Rightarrow(i v)$ These implications are clear.

(iv) $\Rightarrow$ (i) Writing $J=\$ \mathbf{P} \oplus H$, it is enough to show that, if the unital representation $\psi$ of $J$ on $K$ is contractive, then for any $\eta$ in $H$ and all $k_{1}, k_{2}$ in $K$, we have

$$
\left(\psi(\eta)\left(k_{1}\right) \mid k_{2}\right)=-\left(k_{1} \mid \psi(\eta)\left(k_{2}\right)\right)
$$

or equivalently $(\psi(\eta)(k)] k)=0$ for all norm-one element $k$ in $K$. But, denoting by $\alpha$ any positive number, we have

$$
\begin{aligned}
& (\psi(\eta)(k) \mid k)=\frac{((I+\alpha \psi(\eta))(k) \mid k)-1}{\alpha} \leq \frac{\|I+\alpha \psi(\eta)\|-1}{\alpha}= \\
& =\frac{\|\psi(\mathbf{1}+\alpha \eta)\|-1}{\alpha} \leq \frac{\|\mathbf{1}+\alpha \eta\|-1}{\alpha}=\frac{\left(1+\alpha^{2}\|\eta\|^{2}\right)^{1 / 2}-1}{\alpha}
\end{aligned}
$$

(where for the second inequality we have used the assumption that $\psi$ is contractive). Therefore

$$
(\psi(\eta)(k) \mid k) \leq \lim _{\alpha \rightarrow 0+} \frac{\left(1+\alpha^{2}\|\eta\|^{2}\right)^{1 / 2}-1}{\alpha}=0
$$

and, changing $\eta$ by $-\eta$, we obtain $(\psi(\eta)(k) \mid k)=0$.

Now we state and conclude the prof of the main result in this section.

Theorem 2. If $J$ is a smooth nomed Jordan algebra and $\psi$ is a wnital *-representation of $J$ on the pre-Hilbert space of $J$, then the normed space of $J$ with product $\odot$ defined by

$$
x \odot y:=\psi(x)(y)
$$

is an (automatically left division) absolute valued algebra with left unit. Moreover, up to isometric isomorphism, by means of this constructive method all absolute valued algebras with left wit arise.

Proof: The verification of the first paragrapls in the statement is very easy. Since $\psi$ is a unital representation, the unit 1 of 7 as a Jordan 
algebra becomes a left unit for the product $\odot$, and, being also $\psi$ a $*-$ representation, the fact that $J$ with product $\odot$ is absolute valued is a direct consequence of the implication $(i) \Rightarrow(i i)$ in Lemma 2 . Concerning the proof of the second paragraph in the theorem, let $A$ be an arbitrary absolute valued algebra with left unit, so that by Theorem 1 the set

$$
J:=\left\{L_{a}: a \in A\right\}
$$

is a smooth normed Jordan algebra (of bounded linear operators on the normed space of $A$ ). Since $A$ is an absolute valued algebra, the mapping $u: a \rightarrow L_{a}$ is a linear isometry from the normed space of $A$ onto the one of $J$ and, as a consequence, the mapping $\psi: F \rightarrow$ $u \mathrm{Fu}^{-1}$ is a unital isometric (hence $*-$, by the implication $(i i i) \Rightarrow(i)$ in Lemma 2) representation of $J$ on the pre-Hilbert space of $J$. The prool will be concluded by showing that $A$ is isometrically isomorphic to the absolute valued algebra obtained from the pair $(J, \psi)$ by the constructive method in the first paragraph. But the above considered surjective linear isornetry $u: A \rightarrow J$ is also an isomorphism from $A$ onto $(J, \odot)$, because for $a$ and $b$ in $A$ we have

$$
u(a) \odot u(b)=\psi(u(a))(u(b))=u L_{a} u^{-1}(u(b))=u\left(L_{a}(b)\right)=u(a b) .
$$

Remarks 4. i) Most of the information given by the above theorem can be stated without involving Jordan algebras and their representitions on vector spaces, as follows. The norm of any absolute valued algebra $A$ with left unit e derives from an inner product (. . .), and, for $a, b, c$ in $A$ with a orthogonal to e, we have

$$
(a b \mid c)=-(b \mid a c) \text { and } a(a b)=-\|a\|^{2} b .
$$

ii) The Albert-Urbartik-Wright theorem on absolute valued algebras with unit can be easily derived from the above remark. For, if $A$ is such an algebra and 1 denotes its unit element, taking $b=1$ in the last equality we obtain $a^{2}=-\|a\|^{2} 1$ for all $a$ in $A$ othogonal to 1 , hence $A$ is a quadratic algebra. Moreover the same equality now yields to $L_{a}^{2}=L_{a^{2}}$ for $a$ in $A$ orthogonal to 1 , and by symmetry we have also $R_{a}^{2}=R_{a^{2}}$, hence $A$ is alternative. Now $A$ is a division quadratic; alternative algebra, so it is isomorphic to $\mathbb{R}, \mathbb{C}, \mathrm{H}$, or $\bigcirc$ by the extended Frobenius theorem (sce for example [12, Theorem 2.26]).

iii) The examples in Remark 3(i) show obstensibly that isomorphisms between absolute valued algebras can fail to be isometric or even continuous, a pathology that, as we will show in Section 4, only can occur in absence of completeness. However, in the particular case of left-division absolute valued algebras it is not difficult to derive from Proposition 4 and Theorern 2 that isomorphisms must be isonietric. 


\section{Existence of one-sided division absolute valued algebras}

By Corollary 3 the completion of a left division absolute valued algebra is a left division absolute valued algebra, and by Proposition 4 and Theorem 2 every left division complete absolute valued algebra is a Hilbert space. Then one can ask naturally for those cardinal numbers $\aleph$ for which there exist left division complete absolute valued algebras with hilbertian dimension equal $N$. Since in finite dimension the answer is clearly $K=1,2,4$, or 8 (see Proposition 3), we will center our attention in the infinite-dimensional cuse, and in fact we will prove the existence of (antomatically left division) complete absolute valued algebras with left unit of arbitrary infinite hilbertian dimension and with the additional property that they have no nonzero proper closed left ideals (note that every left division algebra has no nonzero proper right ideals). By invoking Theorem 2, the verification of this fact is equivalent to prove that every infinite-dimensional smooth complete normed Jordan algebra has an "irreducible" unital *-representation on its own Hilbert space. At this respect we recall that a self-adjoint set $S$ of bounded linear operators on a real or complex Hilbert space $K$ is said to act irreducibly on $K$ if the only closed $S$-invariant subspaces of $K$ are 0 and $K$. While for complex $K$ this concept has been widely studied, this is not the case for the real context in which we are mainly interested, so we begin our argument with the following

Lemma 3. Let $K$ be a complex Hilbert space, $S$ a self-adjoint subset of $B L(K)$ acting imeducibly on $K$, and $Q$ be any nonzero proper $S$-invariant closed real subspace of $K$. Then $K=Q \oplus^{l_{2}} i Q$. As a consequence, $S$ (regarded as a self-adjoint set of bounded linear operators on the real Hilbert space $Q$ ) acts irreducibly on $Q$.

Proof: First note that, being $Q \cap i Q$ a complex proper closed $S$ invariant subspace of $K$ and acting $S$ irreducibly on $K$, we must have $Q \cap i Q=0$. Denoting by $\pi$ the (real linear) orthogonal projection from $K$ onto $Q$, by the $S$-invariance of $Q$ and the self-adjointness of $S, \pi$ commutes with every element in $S$. Now, regarding complex numbers as linear operators on $K, \pi-i \pi i$ is a bounded complex-linear operator on $K$ commuting with the elements of $S$, hence, by the irreducibility of $S$ on $K$, we have

$$
\pi-i \pi i=\alpha+i \beta
$$

for suitable $\alpha$ and $\beta$ in $\mathbb{R}$ (see $\{7$, Proposition 2.3.8)\}. Multiplying on the right this equality by $\pi$, we have $\pi-i \pi i \pi=\alpha \pi+i \beta \pi$ so, since $Q \neq 0$ 
and $Q \cap i Q=0$, we obtain $\alpha=1$ and $-\pi i \pi=\beta \pi$. By taking adjoints in the last equality it follows that $\beta=0$ and therefore

$$
\pi-i \pi i=1 \text {. }
$$

Thus, since $-i \pi i$ is clearly the orthogonal projection from $K$ onto $i Q$, we have that $i Q$ is the orthogonal complement of $Q$ in $K$, that is $K=$ $Q \Theta^{l_{2}} i Q$. For the consequence assertcd in the statement, note that any nonzero $S$-invariant closed (real) subspace $R$ of $Q$ is a real subspace of $K$ which also satisfies the assumptions on $Q$, hence the above proved fact about $Q$ applies to $R$ giving clearly $R=Q$, and certainly $S$ acts irreducibly on $Q$, as desired.

The existence of "irreducible" unitial *-representations of smooth complete normed Jordan algebras on (real) Hilbert spaces will follow from the above lemma and the next proposition, which contains basic facts about the "canonical articommutation relations", and is taken almost literally from [8, pp. 6-11] (see precisely [8, Proposition 5.2.2]).

Proposition 5. Given a complex Hilbert space $H$, there are a nonzero complex Hilbert space $K$ (the so called Fermi-Fock space of $H$ ) and a conjugate linear mapping $f \rightarrow a(f)$ (the "annithilation" operator) from $H$ into $B L(K)$ satisfying the following three properties:

i) $2 a(f) \cdot a(g)^{*}=(f \mid g) I$ and $a(f), a(g)=0$ for all $f, g$ in $H$ ("canonical anticommutation relations"), where "." denotes Jordan product and $I$ denotes the identity operator on $K$.

ii) $K$ is finite dimensional whenever $H$ is so, while, if $H$ is infinitedimensional, the hilbertian dimension of $K$ equals the one of $H$.

iii) The self-adjoint set of operators $\left\{a(f), a(g)^{*}: f, g \in H\right\}$ acts ireducibly on $K$.

Call a representation of a Jordan algebra on a nonzero Hilbert space irreducible if its range is a self-adjoint set of bounded linear operators acting irreducibly on the given Hilbert space.

Proposition 6. Every smooth complete normed Jordan algebra has an irreducible unital *-representation on a (real) Hilbert space. Moreover for such an algebra (say $J$ ) the following assertions are equivalent:

i) J has an irreducible unital *-representation on its own Hilbert space.

ii) $J$ has a unital *-representation on its own Hilbert space.

iii) The hilbertian dimension of $J$ equals $1,2,4,8$, or any infinite cardinal number. 
Proof: For any cardinal number $\aleph$, consider the complex Hilbert space of (complex) hilbertian dimension $\aleph$, let $K$ and a be respectively the complex Hilbert space and the conjugate-linear mapping from $H$ into $B L(K)$ given by Proposition 5 , and define a (real-linear) mapping $s$ from $H$ into $B L(K)$ by $s(f):=i\left(a(f)+a(f)^{*}\right)$. From assertion (i) in Proposition 5 we obtain

$$
s(f) \cdot s(g)=-\operatorname{Re}(f \mid g) M
$$

for all $f$ and $g$ in $H$, so that the set

$$
J:=\{\lambda I+s(f): \lambda \in \mathbb{R}, f \in H\}
$$

is a real Jordan algebra of operators on $K$ that, algebraically considered, is a copy of the smooth complete normed Jordari algebra of (real) hilbertian dimension $2 \aleph+1$ (actually one can see that, when endowed with the operator norm, this copy is even an isometric copy, but this fact is irrelevant for our argument). Moreover, since clearly

$$
s(f)^{*}=-s(f)
$$

for all $f$ in $H, J$ appears unitally *-represented on the real Hilbert space $K_{\mathbb{R}}$ underlying $K$. In a first instance, taking into account that, the smooth complete nomed Jordan algebra of hilbertian dimension $2 N$ can be unitally *-embedded in the one of hibertian dimension $2 \mathrm{~N}+1$ and applying assertion (ii) in Proposition 5, this argument shows that any frite-dimensional smooth normed Jordan algebra can be unitally *-represented on a nonzero finite-dimensional (real) Hibbert space and, to obtain irreducible unital *-representations, it is enough to pass to the restriction of the operabors in the range of the existing representation to a subspace which is minimal anong the nonzero subspaces that are invariant under the range of the given representation (such a mirimal subspace always exists because of the finite dimensionality). Retaking the initial argument in the infinite-dimensional case, $J$ is the smooth complete norned Jordan algebra of arbitrary infinite lilbertian dimension $N(=2 N+1$ in this case $)$, and the identity operator on $J$ is a unital *-representation of $J$ on the Hilbert space $K_{\mathbb{R}}$ which, in view of assertion (ii) in Proposition 5, has also hilbertian dimension equal to $\mathfrak{K}$. If this representation is 110 irreducible, by the definition of $J$ there inust exist a nonzero proper closed real subspace $Q$ of $K$ invariant under the self-adjoint set of bounded complex-linear operators

$$
S:=\{s(f): f \in H\}
$$


But, it follows from the definition of the mapping $s$ and the conjugatelinearity of the mapping a that

$$
a(f)=\frac{s(i f)-i s(f)}{2} \text { and } a(f)^{*}=-\frac{s(i f)+i s(f)}{2}
$$

for all $f$ in $H$. These equalities, together with assertion (iii) in Proposition 5 , show that $S$ acts irreducibly on $K$ (in the complex sense). By Lemma $3, S$ acts irreducibly on $Q$ (in the real sense) that is, the mapping $T \rightarrow T / Q$ from $J$ into $L(Q)$ is an irreducible unital *-representation of $J$ on the real Hilbert space $Q$ which, in view of the equality $K=Q \oplus i Q$ (see again Lemma 3) and the infinite-dimersionality of $K$, is also of hilbertian dimension equal to $\aleph$. Thus, in any case, the infinite-dimensional smooth complete normed Jordan algebra $J$ of hilbertian dimension $\$$ has an irreducible unital *-representation on a Hilbert space of hilbcrtian dimension $\aleph$. Since the finite-dimensional case has been considered previously, this concludes the proof of the first paragraph in the proposition, and even proves the implication (iii) $\Rightarrow(i)$ in the infinite-dimensional context. To finish the proof of this implication note that, if for $i=1,2,4,8$ we denote by $A_{i}$ the absolute valued algebra $\mathbb{R}, \mathbb{C}, \mathbb{H}, \mathbb{O}$ respectively, the smooth normed Jordan alyebra $J_{i}$ of dimension $i$ can be recognized as the Jordan algebra of operators on $A_{i}$ given by $\left\{L_{a_{i}}: a_{i} \in A_{i}\right\}$, and then the identity mapping on $J_{i}$ is an irreducible unital *-representation on the Hilbert space of $A_{i}$ which of course has dimension $i$. Since the implication $(i) \Rightarrow$ (ii) is clear, let us conclude the proof of the proposition showing that $(i i) \Rightarrow$ (iii), namely, if a smooth complete normed Jordan algebra $J$ has a unital *-representation on its Hilbert space, any finite dimension different from $i=1,2,4,8$ must be cxcluded for $J$. But this follows from the first paragraph in Theorem 2 together with the implication $(i i i) \Rightarrow(i i)$ in Proposition 3 .

With Proposition 4 and Theorem 2, the above proposition leads directly to the following

Theorem 3. Left division complete absolute valued algebras of hilbertian dimension $\mathrm{N}$ exist if and only if $\mathrm{N}$ equals $1,2,4,8$, or any infinite cardinal number. Moreover, for such a cardinal $\aleph$ there exist in fact (antomatically left division) complete absolute valued algebras with left unit of hilbertian dimension $\mathfrak{k}$ with the property that they have no nonzero proper closed left ideals.

Remarks 5. i) Since the completion of a srnooth normed Jordan algebra is a smooth normed Jordan algebra, it follows from the first paragraph in Proposition 6 that every smooth normed Jordan algebra 
has a unital *-representation on a Hilbert space. With the implication (i) $\Rightarrow$ (ii) in Lemrna 2, this shows that every smooth normed Jordan algebra can be viewed as a subspace $P$ of bounded linear operators on a suitable norned space (which actually can be chosen to be a lilbert. space) satisfying the assurnptions in Theorem I. Now certainly we are sure that Theorem 1 camol say more.

ii) Every infinite-dimensional smooth complete normed Jordau algebra $J$ has nonirreducible unital *-representations on its Hitbert spacc. For, if $\psi$ is any unital $*$-representation of $J$ on its Hilbert space, life mapping $\psi \oplus \psi$ from $I$ into $L\left(J \oplus^{l_{2}} J\right)$, given by

$$
\psi \ominus \psi(x)(y, z)=(\psi(x)(y), \psi(x)(z))
$$

for all $x, y, z$ in $J$, is a nonirreducible unital *-representation of $J$ on the Hilbert space $J \mathbb{C}^{l_{2}} J$ which thas the same hilbertian dimension that of $J$. Via Theorem 2, this fact reflects on the existence of complete absolute valued algebras with left unit (of arbitrary infinite hilbertian dimension) having nonzero proper closed left ideals.

iii) If $A$ is an infinite-dimensional left-division absolute valued algebra, then all operators of right multiplication on $A$ are noninvertible. This follows from implication $(i) \Rightarrow(i$ ii $)$ in Proposition 3 .

The rest of this section will be devoted to obtain some interesting consequences of the existence of infinite-dimensional one-sided division absolute valued algebras. The first result we will prove in this direction is that small pertubations of the product of a left division complete absolute valued algebra give rise to new left division algebras, thus providing in view of 'l'heorem 3 a very wide collection of infinite-dimensional complete normed left division algebras. The arbitrarily of the perturbation, together with the structure theory of left division absolute valued algebras (Proposition 4 and Theorem 3), slows that the algebras obtained by this procedure cannot be in general absolute valued algebras. As a matter of fact, we will realize that all these algebras fail to be division algebras.

Proposition 7. Let $A$ be a left division complete absolute valued algebra, let $\square$ be any continuous bilinear prodact on the Banach space of $A$, and let d denote the distance from $\square$ to the product of $A$. Then we have:

i) If $d<1$, the vector space of $A$ with the product $\square$ is a left division algebra.

ii) If $A$ is infinite-dimensional and $d \leq 1$, then all operators of right multiplication on $A$ relative to the product $\square$ are noninvertible. 
Proof: For $a$ in $A$, let us denote as usual by $L_{a}$ and $R_{a}$ respectively the operators of left and right multiplication by $a$ relative to the initial product, and by $L_{a}^{\square}$ and $R_{a}^{\square}$ the ones relative to the product $\square$. If $d<1$, since $A$ is a left division absolute valued algebra, for every $a$ in $A \backslash\{0\}$ we have that $L_{a}$ is invertible and

$$
\left\|L_{a}-L_{a}^{\natural}\right\| \leq d\|a\|<\|a\|=\left\|L_{a}^{-1}\right\|^{-1},
$$

so by completeness of $A L_{a}^{\square}$ is invertible, and (i) is proved. Let us assume $A$ infinite-dimensional and $d \leq 1$. Then, for $a$ in $A$ with $\|a\|=1, R_{a}$ is a linear isornetry form $A$ into $A$ which is not onto (see Remark 5 (iii)) and

$$
\left\|R_{a}-R_{\alpha}^{\square}\right\| \leq d \hat{\|} \mid \underline{\|} 1,
$$

so Lemma 1, together with the fact that the set of noninvertible elements of a Banach algebra is closed, gives that $R_{\alpha}^{\square}$ is not invertible, and (ii) follows.

Given an algebra $A$ and an element $\lambda$ in the base field, the $\lambda$-mutation of $A$, denoted by $A^{(\lambda)}$, is defined as the algebra with the same vector space that of $A$ and product given by

$$
(a, b) \longrightarrow \lambda a b+(1-\lambda) b a .
$$

Corollary 4. Let $A$ be a left division complete absolute valued algebra, and $\lambda$ be a real number. Then we have:

i) If $\lambda>\frac{1}{2}$, then $A^{(\lambda)}$ is a left division algebra and, if in addition $A$ is assumed to be infinite-dimensional, then all operators of right multiplication on $A^{(\lambda)}$ are noninvertible.

ii) If $\lambda=\frac{1}{2}$ and $A$ is infinite-dimensional, then all operators of left (=right) nultiplication on $A^{(\lambda)}$ are noninvertible.

iii) If $\lambda<\frac{1}{2}$, then $A^{(\lambda)}$ is a righl division algebra and, if in addition $A$ is assumed to be infinite-dimensional, then all operators of left multiplication on $A^{(\lambda)}$ are noninvertible.

Proof: (i) and (ii) follow directly from Proposition 7 by taking as the product $\square$ the one given by

$$
a \square b:=a b+\frac{1-\lambda}{\lambda} b a,
$$

while (iii) follows from (i) applied to $1-\lambda$ taking into account that the opposite algebra of $A^{(1-\lambda)}$ is $A^{(\lambda)}$. 
Remark 6. A consequence of the above corollary is the well-known fact that, all $\lambda$-mutations with $\lambda \neq \frac{1}{2}$ of a finite-dimensional absolute valued algebra are division algebras.

Our concluding result in this section, together with Theorem 3, will show the existence of infinite-dimensional complete normed algebras such that the operators of left and right multiplication by any nonzero element are surjective. Unfortunately, in our examples all these operators will fail to be one-to-one. Our argument begins with the easy observation in the following lemma. By involution on a real (resp.: complex) vector space we mean a linear (resp: conjugate-linear) operator on the space with square the identity operator.

Lemma 4. Let $A$ be a real or complex complete absolule valued algebra whose Banach space is a Hilbert space, let - be an involution on the vector space of $A$, and define a new (bilinear) product $\square$ on $A$ by

$$
a \square b:=R_{\bar{b}}^{*}(a)
$$

(where, as usual, $R_{b}$, denotes the operator of right multiplication by $b$ relative to the initial product). Then, for every $b$ in $A \backslash\{0\}$, the operator $R_{b}^{\square}$ of right multiplication by b relative to the product $\square$ is surjective.

Proof: Note that $R_{b}^{\square}=R_{\bar{b}}^{*}$, so it is enough to prove tha $R_{b}^{*}$ is a surjective operator for any norm-one element $b$ in $A$. But, being $A$ an absolute valued algebra, for such a $b, R_{b}$ is a linear isometry so, since the Banach space of $A$ is a Hibert space, we have $R_{b}^{*} R_{b}=I$ (the identity operator on $A$ ), lience certrainly $R_{b}^{*}$ is surjective.

Our following observation, which is also of easy verification, involves standard terminology of $H^{*}$-algebras. Following [23] and [11]; a left semi- $H^{*}$-algebra will be a real or complex Hilbert space $A$ together with a continuous bilinear product on $A$ (denoted usually by juxtaposition) and an involution - on the vector space of $A$ satisfying

$$
(a b \mid c)=(b \mid \bar{a} c)
$$

for all $a, b, c$ in $A$.

Lemma 5. Let $A$ be a real or complex left semi-H*-algebra whose involution - is isonetric, and defne a new product $\square$ on $A$ by $a \square b:=$ $K_{\bar{b}}^{*}(a)$. Then - is an algebra involution on $(A, \square)$ (that is, $\overline{a \square b}=\bar{b} \square i b$ for all $a, b$ in $A$ ). 
Proof: By definition of the product $\square$, we have

$$
(a \square b \mid c)=(a \mid c \bar{b})
$$

for all $a, b, c$ in $\mathrm{A}$. Using this fact, together with the axiom of left serni$H^{*}$-algebras

$$
(a b \mid c)=(b \mid \bar{a} c)
$$

and the assumed isometry of the involution

$$
(a \mid b)=(\bar{b} \mid \bar{a})
$$

we obtain for arbitrary $a, b, c$ in $A$

$$
\begin{aligned}
(\bar{b} \square \bar{a} \mid c) & =(\bar{b} \mid c a)=(\bar{c} \bar{b} \mid a)=\overline{(a \mid \bar{c} \bar{b})}= \\
& =\overline{(a \square b \mid \bar{c})}=(\bar{c} \mid a \square b)=(\overline{a \square b} \mid c),
\end{aligned}
$$

hence $\overline{a \square b}=\bar{b} \square \bar{a}$, as required.

Proposition 8. Let $A$ be a complete absolute valued algebra with left unit $e$. Let ${ }^{-}$denote the unique isometric linear involution on the Hilbert space of $A$ having Re as the set of fixed points, and define a new product $\square$ on $A$ by $a \square b:=R_{i}^{*}(a)$. Then we have:

i) $(A, \square)$ is a conplete nomned algebra on which - becomes an algebra involution.

ii) The operators of left and right multiplication by any nonzero element on $(A, \square)$ are surjective.

iii) For any $a$ in $A$, the equalities $a \square \bar{a}=\bar{a} \square a=\|a\|^{2}$ e hold.

Proof: Clearly the mapping $\lambda e+q \rightarrow \lambda e-q$ from $A=\mathbb{R e} \oplus^{\ell_{2}} Q(Q$ denoting the orthogonal complement of $\mathbb{R} e$ ) into $A$ is the only isometric involution on the Hilbert space of $A$ having exactly as fixed points the clements of $\mathbb{R} e$. Also with this involution (call it, as in the statement of the proposition) $A$ becomes a left semi- $H^{*}$-algebra (sce Remark 4(i)). Now the nortrivial part of assertion (i) follows from Lemma 5 , while (ii) follows from (i) and Lemma 4. To prove (iii), note that for every $a$ in $A$ we have $R_{a}^{*} R_{12}=\|a\|^{2} I$ (see the proof of Lemma 4). When applied to $e$ this equality gives

$$
a \square \bar{a}=\|a\|^{2} e,
$$

and the remaining equality $\bar{a} \square a=\|a\|^{2} e$ follows from the isometry of 
Remark 7. With the notation in the above proposition, assurne that $A$ is infinite-dimensional. Then it is easy to see, using Remark 5(iii), that no operator of left or right multiplication on $(A, \square)$ is one-to-one.

\section{Homomorphisms into absolute valued algebras}

Some results in Sections 1 and 2 will be applied here to obtain that homomorphisms from complcte normed algebras into arbitrary absolute valued algebras are contractive, hence continuous. The proof of this result we will give also involves some arguments in [21], and begins with the following

Lemma 6. Let $X$ and $Y$ be Banach spaces, $\Phi$ a linear mapping from $X$ into $Y$ with dense range, $F$ a bounded linear operator on $X$, $G: Y \rightarrow Y$ a nonsurjeclive linear isometry, and assume $\Phi F=G \Phi$. Then $\|F\| \geq 1$.

Proof: Iet $\lambda$ be in $\mathbb{R}$ with $|\lambda|<1$, so that $\|G-(G-\lambda I)\|<1$. By Lemma 1 , the range of $G-\lambda I$ is a proper closed subspace of $Y$. Since

$$
\Phi(F-\lambda I)=(G-\lambda I) \Phi
$$

and $\Phi$ has dense range, it follows that $F-\lambda I$ cannot be surjective, so $F-\lambda I$ cannot be invertible in the Banach algebra $B L(X)$, and so $|\lambda| \leq \mid i F \|$. Now the proof is concluded by letting $\lambda \rightarrow 1$.

Theorem 4. Let $A$ be a complete normed algebra, $B$ an absolute valued algebra, and $\Phi$ be a homomorphism from $A$ into $B$. Then $\Phi$ is contractive.

Proof: Regarding $\Phi$ as a mapping from $A$ into the completion of its range, we may assume that the absolute valued algebra $B$ is complete and that the homomorphism $\Phi$ has dense range. First assume additionally that $\Phi$ is actually surjective, so that, since the equality

$$
\Phi L_{a}=L_{\Phi(a)} \Phi
$$

holds for arbitrary $a$ in $A$, we can apply $[\mathbf{2 1}$, Lemma 3.1$]$ to obtain

$$
r\left(L_{\Phi(a)}\right) \leq r\left(L_{a}\right)\left(\leq\left\|L_{a}\right\| \leq\|a\|\right)
$$

(where $r$ denotes spectral radius, $r(T):=\lim _{n \rightarrow \infty}\left\|T^{n}\right\|^{1 / n}$ ). The result in this first considered case now follows from the clear fact that 
$r\left(L_{b}\right)=\|b\|$ for all $b$ in any absolute valued algebra. Consider the remaining case of $\Phi$ being not onto. Then, since $\Phi$ has dense range, $B$ must be infinite-dimensional, and then the implication (i) $\Rightarrow(i i i)$ in Proposition 3 gives that, if some left multiplication operator on $B$ is invertible, then all right multiplication, operators on $B$ are noninvertible, hence (changing $A$ and $B$ by their corresponding opposite algebras, if necessary) we may assume that all left multiplication operators on $B$ are noninvertible. To conclude the proof it is enough to show that $\|a\| \geq 1$ whenever $a$ is in $A$ with $\|\Phi(a)\|=1$. But, for such an $a, L_{\Phi(a)}$ is a nonsurjective linear isometry, hence, by $\left(^{*}\right)$ and Lemma 6 , we deduce

$$
1 \leq\left\|L_{a}\right\|(\leq\|a\|)
$$

as desired.

Acknowledgements. This paper has benefited from several interesting suggestions by M. Cabrera, J. A. Cuenca, R. Payá, J. Pérez, J. Martínez, W. Werner, and E. Zel'manov. The author is very grateful to them.

\section{References}

1. A. A. AlBert, Absolute valued algebras, Ann. Math. 48 (1947), 495-501.

2. A. A. AfBert, Absolute valued algebraic algebras, Bull. Amer. Math. Soc. 55 (1949), 763-768. A note of correction, Ibid. 55 (1949), 1191.

3. S. F.. Bellenot, Banach. $S$-algebras and conditional basic sequences in non-Montel Fréchet spaces, Studia Math. 79 (1984), $17-33$.

4. F. F. BONSALl AND J. DUNCAN, "Complete normed algebtas," Springer-Verlag; 1973.

5. F. F. BONSALI AND J. DUNCAN, "Numerical ranges $I_{\text {," London }}$ Math. Soc. Lecture Note Series 10, Cambridge University Press; 1973.

6. R. BOTT AND J. MILNOR, On the parallelizability of the spheres; Bull. Amer. Math. Soc. 64 (1958), 87-89.

7. O. BRATTELJ AND D. W. RoBINSON, "Operator algebras and quantum statistical mechanics I,". Springer-Verlag, 1979. 
8. O. BRATtEli AND D. W. ROBINSON, "Operator algebras and quantum statistical mechanics II," Springer-Verlag, 1981.

9. M. Cabrera and A. Rodríguez, Nonassocialive ultraprime nomed algebras, Quart. J. Math. Oxfond 43 (1992), 1-7.

10. J. A. CUEnCA, One-sided division infinite-dimensional normed real algebras, Publicacions Malemàtiques 36 (1992), 485-488.

11. J. A. Cuench and A. Rodríguez, Stracture theory for noncommutative Jordan $H^{*}$-algebras, J. Algebra 106 (1987), 1-14.

12. H. HANCHE-OLSEN AND E. STøRMER, "Jordan operator algebras," Pitman, 1984.

13. N. JACOBSON, "Structure and representation of Jordan algebras," Amer. Math. Soc. Coll. Publ. 39, Providence, Rhode Island, 1968.

14. A. M. KAIDí, Bases para una teoría de las álgebras no asociativas normadas, 'Tesis Doctoral, Universidad de Granada (1978).

15. M. L. EL-MALLAH, Sur les algèbres absolument valućes qui vérifient l'identité $(x, x, x)=0, J$. Algebra 80 (1983), 314-322.

16. M. L. El-MALLAH, On finite dimensional absolute valued algebras satisfying $(x, x, x)=0$, Arch. Math. 49 (1987), 16. 22.

17. M. L. El-MaLLAH, Absolute valued algebras with an involution, Arch. Math. 51 (1988), 39-49.

18. M. L. EL-MALLAH, Absolute valued algebras containing a central idempotent, J. Algebro 128 (1990), 180-187.

19. M. L. Er-MAI,IAH AND A. MiCAri, Sur les dimensions des algèbres absolument valuées; $J$. Algebra 68 (1981), 237-246.

20. A. Rodríguez, Nonassociative normed algebras spanned by hermitian elements. Proc. London Math. Soc. 47 (1983), $258 \cdot 274$.

21. A. Ronníguez. The uniqueness of the complete norm topology in complete normed nonassociative algebras, J. Funct. Analysis 60 (1985), 1-15.

22. A. Rodríguez, An approach to Jordan Banach algebras from the theory of nonassociative complete normed algebras, Ann. Sci. Univ. "Blaise Pascal", Clermont II, Sér. Math. 27 ème (1991), 1-57.

23. M. F, Smuley, Right $H^{*}$-algebras, Proc. Amer. Math. Soc. 4 (1953), 1-4.

24. K. Unbank, Absolute valued algebras with an involution, Fandamenta Math. 49 (1961), 247-258.

25. K. URBANIK, Reversibility in absolute valued algebras, Fundamenta Math. 51 (1962), 131-140.

26. K. Urbanik and I². B. Wricht, Absolute valued algebras, Proc. 
Amer. Math. Soc. 11 (1960), 861-866.

27. F. B. Wright, Absolute valued algebras, Proc. Nat. Acad. Sci. U.S.A. 39 (1953), 330-332.

Departamento de Análisis Matemático

Facultad de Ciencias

Universidad de Granada

1S071-Granada

SPAIN

Rebut el 7 de Gener de 1992 